\title{
CONTROL DE UN ROBOT MÓVIL MEDIANTE INTERFAZ CEREBRO-COMPUTADOR: UN ENFOQUE PRÁCTICO.
}

\author{
R. López de Ahumada, G. Amitrano *, F. Gómez-Bravo, R. Jiménez Naharro, J. A. Gómez Galán \\ Depto. Ingeniería Electrónica, Sistemas Informáticos y Automática. \\ Escuela Técnica Superior de Ingeniería, \\ Universidad de Huelva. Carretera de Palos-La Rábida s/n. 21071 Huelva. \\ \{ ahumada ;fernando.gomez; naharro; jgalan \}@diesia.uhu.es \\ * Alten Italia. Via Gaetano Crespi, 12 Milano 20134, Italia \\ gianluca.amitrano@alten.it
}

\section{Resumen}

Este trabajo muestra un ejemplo de la interacción entre ondas cerebrales y plataformas robóticas. Concretamente se realiza el control de un robot móvil mediante el dispositivo llamado Mindwave, producido por la compañía Neurosky. Dicho dispositivo, a pesar de poderse considerar en la categoría de bajo precio, es capaz de proporcionar los niveles de atención, entre otros, de un usuario a partir de la medida de las señales eléctricas generadas por su cerebro. La plataforma desarrollada se basa en un software diseñado en el entorno Matlab, para identificar las intenciones del usuario y transmitir las órdenes correspondientes al robot. Los resultados de las pruebas realizadas son diferentes a los reportados en la literatura, logrando una aplicación práctica, que consigue reducir los tiempos de retardo y requiere un nivel de entrenamiento reducido. Se presentan resultados de una simulación virtual. Dichos resultados han sido corroborados mediante experimentos reales.

Palabras Clave: Robots móviles; Teleoperación; Sistemas ICC.

\section{INTRODUCCIÓN}

El estudio de las Interfaces Cerebro-Computador (ICC o BCI de las siglas en inglés Brain-Computer Interface) se ha desarrollado enormemente en la última década. Desde entonces se han implementado varias aplicaciones utilizando diferentes dispositivos que son capaces de capturar las señales eléctricas producidas por la interacción de las neuronas del cerebro humano [2][4][5][8]. Durante estos últimos años, se han utilizado numerosas herramientas software que permiten interconectar dispositivos ICC con computadores [1]. Gracias a ellas, muchas personas han sido sometidas a muy diversas pruebas mientras que estaban en diferentes estados de conciencia. Además, mediante su uso, los investigadores han intentado controlar varios tipos de plataformas robóticas, desde aplicaciones básicas (como simulaciones virtuales, control del puntero del ratón, etc.) hasta aplicaciones más complejas en la que se intenta controlar de la mejor manera posible robots, manipuladores o sillas de ruedas. La posibilidad de comunicación cerebro-computador basado en el electroencefalograma (EEG) se discutió hace casi cuatro décadas.

Aunque hasta los inicios de este siglo no había más de 5 grupos de investigación activos en este campo, hoy día varias centenas de laboratorios trabajan sobre el tema. Este espectacular crecimiento ha sido impulsado por un alto rendimiento y un bajo coste de la potencia de cálculo y la instrumentación relacionada, permitiendo alcanzar mayor comprensión sobre las funciones cerebrales normales y anormales y mejorar los métodos para descifrar señales cerebrales en tiempo real. Como resultado, el rendimiento y la usabilidad de los sistemas ICC han avanzado considerablemente en los últimos años, la importancia de las propiedades específicas han cambiado y nuevas tecnologías se han desarrollado permitiendo nuevas aplicaciones y haciendo los sistemas ICC más asequibles. Por ejemplo, a finales de los años 90 había sólo unos pocos sistemas en tiempo real en todo el mundo. En la actualidad, es incontable el número de laboratorios que están equipados con sistemas ICC en tiempo real.

Como se describe en [3], para destacar estos avances y desarrollos tecnológicos en el campo de las Interfaces Cerebro-Computador, desde el año 2010 se otorga un premio de carácter anual, reconociendo las investigaciones excepcionales e innovadoras. No es de extrañar que la mayoría de los trabajos desarrollados utilicen sistemas ICC en su mayoría basados en EEG porque son más fáciles de manejar y son más baratos. La aplicación más práctica es sobre todo en la ortografía, por delante de aplicaciones de control general, rehabilitación de accidente cerebro 
vascular, control de silla de ruedas o de robots y control de Internet [1].

En [5] se presenta un sistema ICC para ayudar a personas con discapacidad en el control externo de un robot de servicio. El sistema se basa en el uso de las ondas alfa del Electroencefalograma (EEG). El bloque de ondas alfa se reconoce, procesa y se transmite para controlar una sola opción, mientras que se usa una máquina de estados para un control de múltiples opciones. Los sujetos fueron capaces de utilizar el sistema de manera fiable después de un periodo corto de entrenamiento. En este trabajo, el software utilizado fue desarrollado en LabView siendo una de las partes más importantes del mismo. La opción requerida por el usuario para controlar el robot es seleccionada mediante el cierre de los ojos, lo que produce un cambio en las ondas alfa, que es detectado, cuando un led determinado se enciende en el panel de control. En ese mismo trabajo se constata que la operación en tiempo real introduce un tiempo de retraso de dos minutos, debido a la necesidad de procesar adecuadamente la señal del EEG. Además, debido a que el cambio de dirección se realiza por software, la precisión en la conducción se ve influenciada por la rugosidad de la superficie del suelo, y la propia dirección anterior. Las limitaciones temporales vienen impuestas por la necesidad de seleccionar el movimiento direccional. Si bien el tiempo para activar y seleccionar con el interruptor de la onda alfa no se puede reducir sustancialmente debido a la tecnología de opción múltiple elegida, los autores proponen mejorar los tiempos de selección mediante el establecimiento de una tensión umbral más baja que el valor indicado en este sistema, pero ello conduce a un elevado número de errores.

La metodología que se presenta en [2] combina un método de selección de características y un algoritmo de clasificación probabilístico para construir el modelo predictivo de la intención anticipada de movimiento voluntario de pacientes con temblor a partir de un solo ensayo. Los resultados obtenidos muestran que esta combinación es una buena base para la construcción de ICCs que no requieran entrenamiento del usuario de forma personalizada, asíncrona y adaptativa. Para ello es necesario establecer parámetros óptimos de la señal EEG de forma personalizada, mediante una exhaustiva investigación, uso de un potente algoritmo predictivo y uso de técnicas de minería de datos. Es de destacar que la experimentación del paradigma se hizo con sujetos no sanos. La detección del pre-movimiento permite acortar tiempos de respuesta, aunque en este caso estaba orientada a la corrección de movimientos involuntarios. El sistema debe usar una red amplia y compleja de sensores que puedan monitorizar las ondas cerebrales y encontrar las que proporcionen las pistas suficientes para detectar que se va a realizar un movimiento. La realimentación de información al sistema se hace también con sensores que detectan el movimiento.

En [4], se presenta un trabajo en el que un ICC se usa para tele-operación de dos sistemas robóticos de bajo coste, aunque el elemento importante de adquisición de las señales EEG es una red de sensores de 16 electrodos, de un coste elevado. El objetivo es demostrar la posibilidad de tele-operación de un número arbitrario de estaciones robóticas en pacientes con dificultad motora. Como en muchos casos de control, se usa el denominado protocolo P300, mediante el uso de estímulos visuales y la correspondiente alteración de la señal EEG, lo que permite una efectiva detección a costa de un procesamiento lento de la información. Aunque los robots ejecutan autónomamente los comandos enviados, la selección previa del comando, la transmisión por internet y bluetooth al robot suman un retraso que debe ser tenido en cuenta, ya que aunque no está contabilizado en su totalidad, parece ser superior a 5 segundos.

El software en [8] fue de nuevo desarrollado con LabView. La monitorización del nivel de atención y la cuenta del número de parpadeos de los ojos se hace mediante bloques simples y se usan para programar los diferentes movimientos del robot. El procesamiento de señales que involucran el control del robot es complejo y requiere, además, de cierta intervención humana.

En todos estos trabajos los sistemas ICC empleados están basados en una red sensores compleja y solo manejable por personal cualificado.

En el presente trabajo se ha desarrollado una plataforma que permite teleoperar un robot móvil mediante las señales que proporciona un ICC. El ICC utilizado procesa las ondas cerebrales y caracteriza el nivel de atención o meditación del usuario. Estas señales se complementan con una señal asociada, también generada por el propio ICC, que en este caso es la intensidad con la que se parpadea. Con todo ello, el sistema implementa un método de control que hace posible al usuario controlar un robot mediante el control de su estado de atención y la intensidad de su parpadeo. Respecto a trabajos anteriores se mejora la facilidad con que el usuario aprende a maniobrar el robot (entre unas pocas horas y unos pocos días) y en la mejora del retardo en el procesado de la señal del ICC, y todo ello, se consigue con una tecnología accesible y de fácil adquisición.

El artículo, tras esta introducción presenta en la sección 2 una descripción del sistema y sus componentes. En la sección 3 se detallan los 
pormenores de la estrategia de control seguida. La sección 4 está dedicada a ilustrar los resultados experimentales y describir el método de adiestramiento con el que los usuarios del sistema han aprendido a interaccionar con el sistema. Finalmente el artículo termina con las conclusiones y las referencias.

\section{DESCRIPCIÓN DEL SISTEMA}

En este trabajo se ha pretendido desarrollar una plataforma que permita que una persona, sin mucho entrenamiento ni formación técnica específica, controle de manera sencilla el movimiento de un robot móvil utilizando un ICC accesible (con un costo muy inferior a los sistemas que hasta ahora venían utilizándose). El espíritu que fundamenta su diseño, es el de encontrar una solución práctica y económica que haga posible la teleoperación del robot, sin que sea necesario que el sistema ICC cumpla con elevadas restricciones tecnológicas. Para ello, se han utilizado tres elementos básicos: el ICC MindWave desarrollado por Neurosky [6], una plataforma móvil construida con el kit Mindstorm de Lego, y una aplicación residente en un computador personal.

\subsection{ARQUITECTURA DEL SISTEMA}

La arquitectura del sistema desarrollado se muestra en la Fig. 1. Es de destacar que la conexión entre los tres elementos se realiza de forma inalámbrica, dotando de cierta libertad de movimiento al usuario. El núcleo del mismo es una aplicación desarrollada en Matlab. En ella se interacciona de forma independiente con el ICC y con el robot. Por una parte el software sirve para leer los datos capturados por el ICC y formatearlos correctamente. Por otra, la aplicación implementa un algoritmo de control que facilita la labor de conducción. El usuario tiene una realimentación visual del comportamiento del robot y mediante el control de las señales captadas por el ICC debe ser capaz de mover el robot desde un punto específico a otro inicialmente establecido.

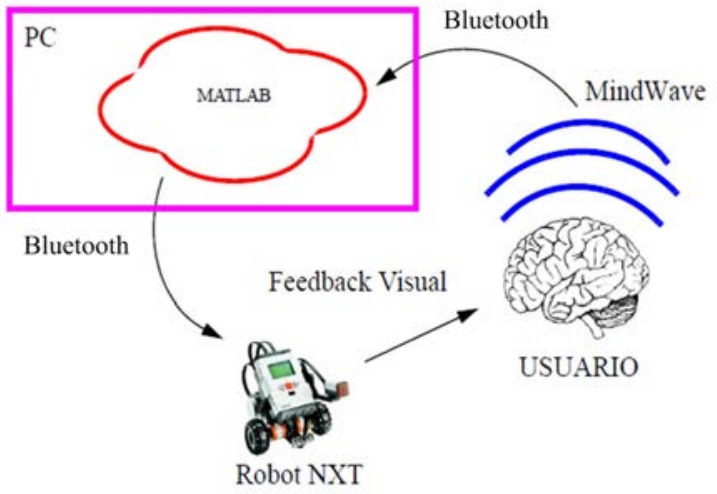

Figura 1: Arquitectura del sistema

\subsection{MINDWAVE DE NEUROSKY}

El sistema ICC de MindWave (Fig. 2) permite estimar el estado de atención y de meditación (entre otros parámetros) de la persona usuaria. Las señales de atención y meditación están establecidas por el comportamiento de determinadas ondas cerebrales. En particular, las ondas alfa son dominantes cuando el nivel de meditación es alto y las ondas beta predominan cuando el nivel de atención es alto. El sistema Mindwave, es capaz de registrar estas ondas y establecer los niveles de atención y meditación utilizando solo dos sensores, lo que representa una ventaja en comparación con otros sistemas más complejos y dificultosos que necesitan más de 24 electrodos. Junto a esta reducción, hay que señalar como ventaja que los electrodos son secos, lo que mejora la precisión en la medición de la onda cerebral.

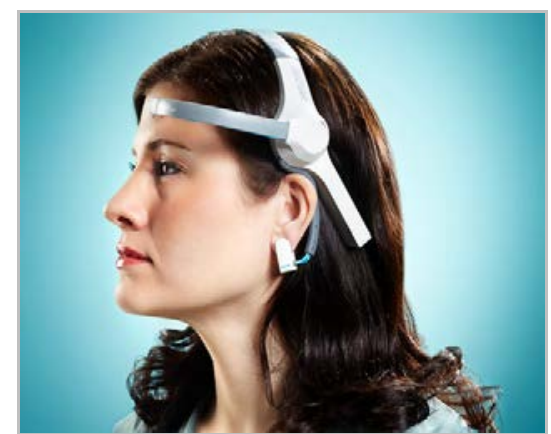

Figura 2: Dispositivo Mindwave de Neurosky

Concretamente, MindWave informa de los estados atención y meditación utilizando los algoritmos eSense de Atención y Meditación, propiedad de NeuroSky, junto con las ondas registradas y la información sobre las bandas de frecuencias de la onda cerebral, que es proporcionada por la tecnología ThinkGear (interna de cada producto de NeuroSky). Dicha tecnología incluye el sensor que toca los puntos de la frente, el contacto y la referencia en el clip de la oreja y el circuito integrado que procesa todos los datos. Las formas de las ondas cerebrales y las mediciones eSense (atención y meditación) se calculan en dicho circuito amplificando la señal de las ondas cerebrales, eliminando el ruido ambiente y el movimiento muscular. El algoritmo eSense se aplica entonces a la señal restante dando como resultado unos valores que se pueden interpretar. Hay que tener en cuenta que los valores de la medida eSense no describen un número exacto sino los rangos de actividad del sujeto. Cada tipo diferente de valor eSense se informa en una escala relativa de 1 a 100. En esta escala, un valor entre 40 a 60 en un determinado momento se considera "neutral" y es similar en concepto a las "líneas de base" que se establecen en las técnicas de medición de las ondas cerebrales convencionales (aunque el método para determinar una línea de base ThinkGear es 
propietario y puede diferir de los métodos convencionales). Un valor de 60 a 80 se considera "ligeramente elevado", y puede ser interpretado como niveles de atención o meditación que pueden ser mayores de lo normal para una persona determinada. Los valores de 80 a 100 se consideran "elevados". Del mismo modo, en el otro extremo de la escala, un valor entre 20 a 40 indica niveles "reducidos", mientras que un valor entre 1 y 20 indica niveles "muy bajos". Estos niveles pueden indicar estados de distracción, agitación, o anomalía.

La razón de disponer de rangos tan amplios de valores para cada posible interpretación es que algunas partes del algoritmo de eSense aprenden de forma dinámica $\mathrm{y}$, a veces se emplean algunos algoritmos de adaptación lentos, de manera que se ajusta a las fluctuaciones y las tendencias de cada usuario de forma natural, lo que representa y compensa el hecho de que las ondas cerebrales en el cerebro humano están sujetas a fluctuaciones. Por ello, los sensores del ThinkGear son capaces de operar con una amplia gama de individuos bajo un extremadamente amplio rango de condiciones personales y ambientales, al tiempo que proporcionan una buena precisión y fiabilidad.

Adicionalmente, el sistema Mindwave es capaz de captar otra señal llamada 'eye blink strength' (intensidad de parpadeo), que informa de la intensidad del parpadeo de ojo del usuario. Su valor va de 1 a 255 y se proporciona cuando se detecta un parpadeo del ojo. El valor indica la intensidad relativa de un parpadeo sin unidades. El sensor de la frente del Mindwave debe estar muy apretado para que no se detecten parpadeos espurios, que generalmente tienen valores de entre 30 y 40 (el mismo valor de un parpadeo de ojos natural).

El sistema Mindwave se comunica con el PC, para la transmisión de los datos, mediante el uso de un adaptador inalámbrico USB específico para este ICC.

\subsection{LEGO MINDSTORMS NXT}

El Lego Mindstorms NXT es un kit de construcción de robots que se puede programar en diferentes lenguajes de programación. El kit contiene tres servomotores, y diversos sistemas sensoriales, cables, batería, y un ladrillo inteligente NXT que constituye la unidad de control principal. El ladrillo capaz de leer los sensores y actuar sobre los motores. Además, éste dispone de un procesador de 8 bits completamente dedicado a la gestión de entradas y salidas del NXT y un pequeño procesador que se encarga de las comunicaciones inalámbricas mediante Bluetooth. Con estos elementos puede construirse un sencillo robot que interactúa con su entorno.
Particularmente, en este trabajo se ha utilizado un robot con una configuración básica de tracción diferencial [7]. La utilización de este tipo de configuración facilita la estrategia de control al permitir desacoplar el control de la velocidad lineal del control de la velocidad de giro del robot. Gracias a esta característica, el movimiento puede: descomponerse en movimientos básicos de desplazamientos lineales y giros, o bien, en el caso de usuarios más avanzados, permite la ejecución de trayectorias continuas más complejas.

\subsection{APLICACIÓN SOBRE MATLAB}

El esquema general de la aplicación obedece al diagrama de flujo que se muestra en la Fig. 3. En él puede observarse cómo tras realizarse la fase de inicialización de variables y configuración de puertos de comunicación, se ejecuta un bucle iterativo que finaliza una vez transcurrido un tiempo determinado. Dentro de este bucle se ejecutan secuencialmente: la lectura de datos del ICC, la evaluación de una máquina de estados que determina las acciones que debe realizar el robot y el envío de las consignas de control al robot NXT. El algoritmo es simple y por ello Matlab puede trabajar a tiempo real.

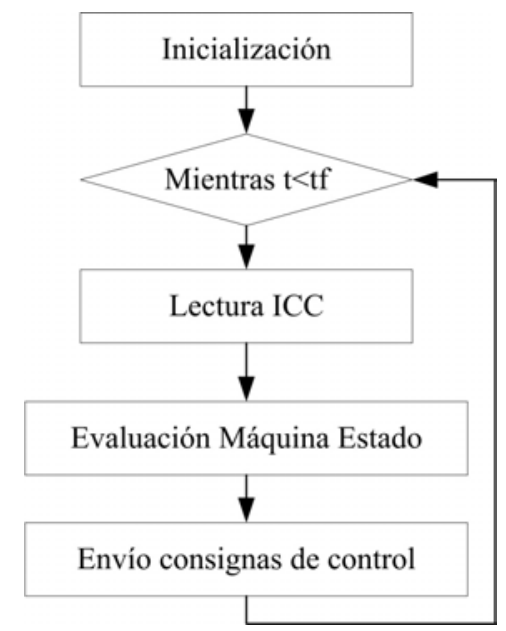

Figura 3: Diagrama de flujo de la aplicación

\subsubsection{Lectura de datos del MindWave}

Los componentes ThinkGear entregan sus datos digitales como una secuencia de bytes asíncrona en serie. Esta secuencia debe analizarse e interpretarse como paquetes ThinkGear para poder extraer los valores de datos correctamente $\mathrm{y}$, mediante software, procesarlos en un computador. Neurosky proporciona una librería específica que ayuda al programador a establecer una comunicación entre el Mindwave y el PC de una manera simple y sencilla. Haciendo uso de dicha librería, es posible acceder desde Matlab al paquete de datos y extraer los valores de atención, meditación e intensidad de parpadeo. 
De forma paralela al diseño de la aplicación para el control del robot, se ha desarrollado una aplicación especial para monitorizar el nivel de las señales y permitir el entrenamiento de los usuarios. Concretamente, la Fig. 4 muestra una gráfica de las señales adquiridas desde el Mindwave y procesadas por Matlab, en tiempo real. El nivel de atención está representado en rojo y el de meditación en azul.

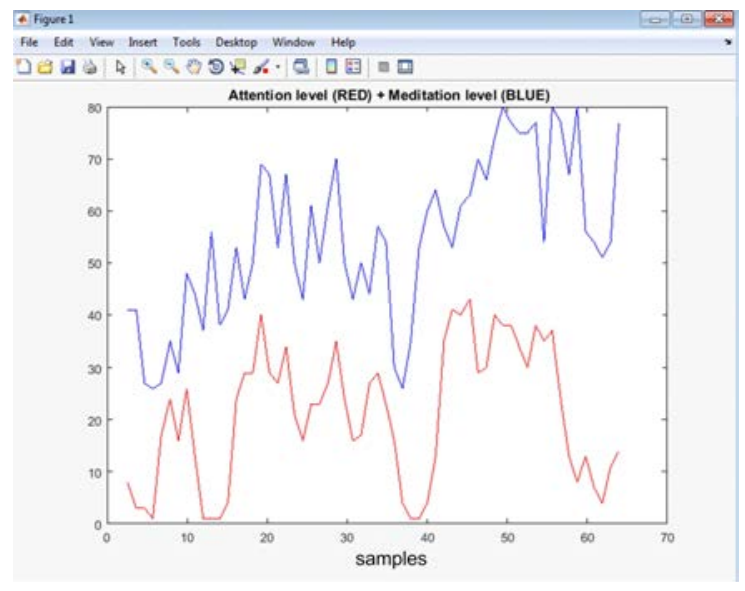

Figura 4: Gráfica de las señales de meditación y atención.

La representación se corresponde con 65 muestras, una por segundo, en la que el nivel de atención está siempre por debajo del de meditación. En ningún momento el usuario intentó adquirir un nivel determinado. Esta aplicación resulta muy útil en el proceso de entrenamiento, permite desarrollar la habilidad de controlar la amplitud de la señal mientras observa la tendencia de la onda.

\subsubsection{Librería de control del Lego Mindstorm}

Para controlar el comportamiento del robot desde Matlab, se ha usado la Toolbox RWTH - Mindstorms NXT, que es un proyecto desarrollado por la Universidad de RWTH Aquisgrán [9]. Este software ha sido desarrollado para controlar el kit de robot mediante Matlab a través de una conexión inalámbrica Bluetooth o por USB. Este software es un producto en código abierto y está sujeto a la licencia pública GNU (GPL). Con este software es posible interactuar con el robot usando comandos del MATLAB a través de Bluetooth.

\subsubsection{El módulo de control}

El peso del control del robot móvil recae en una máquina de estados que toma como valores de entrada los niveles de las señales proporcionadas por el ICC, y evoluciona activando y desactivando un conjunto de estados que están asociados a las distintas formas de moverse que tiene el robot móvil.
Esta máquina de estado junto con la estrategia general de control se ilustra en la siguiente sección.

\section{ESTRATEGIA DE CONTROL}

La estrategia de control principal se basa en el uso de las señales de atención, meditación e intensidad de parpadeo para determinar las intenciones del usuario.

La razón principal para utilizar estas tres señales es que son fácilmente manejables sin un excesivo entrenamiento. Tras muchas pruebas [6], resulta evidente que cada persona tiene una capacidad diferente en el control del nivel atención y meditación: algunas personas pueden meditar fácilmente y otras puede llegar a niveles de atención altos en un tiempo muy corto. Aunque en una primera instancia la idea fue utilizar las señales de meditación y atención a la vez para controlar la plataforma robótica, se comprobó que la mayoría de los usuarios sin gran entrenamiento no son capaces de controlar ambas señales al mismo tiempo (aclarar la mente para estar en un estado de meditación generalmente no ayuda al usuario a alcanzar un estado de atención profundo).

Por este motivo, se incorporó a la estrategia de control el uso de la intensidad de parpadeo. Es posible para un usuario no muy entrenado mantener un determinado nivel de atención o de meditación y controlar al mismo tiempo cambios en la intensidad de parpadeo. De esta forma, la máquina de estado que controla el robot atenderá a los cambios sufridos por dos señales (atención o meditación y parpadeo) para evolucionar de uno a otro estado.

\subsection{LA MÁQUINA DE ESTADOS}

La idea que se presenta en este trabajo consiste en controlar el movimiento del robot mediante dos parámetros, la velocidad lineal $\left(\mathrm{v}_{\mathrm{l}}\right)$ y la velocidad de rotación (w). Aunque pueden utilizarse estrategias más complicadas, las experiencia aquí ilustrada se basa en la conmutación de dos comportamientos: movimiento lineal (ML: $\mathrm{v}_{\mathrm{l}}$ activa $\mathrm{y} \mathrm{w}$ desactiva); movimiento de giro (MG: $\mathrm{v}_{\mathrm{l}}$ desactiva $\mathrm{y} \mathrm{w}$ activa).

Como se mencionó antes, la mayoría de las personas tienen dificultad para controlar las señales de meditación y atención al mismo tiempo, por lo que no es buena idea asociar directamente las variables de control del robot a los niveles de ambas señales.

Por el contrario, la solución adoptada se apoya en la utilización de una máquina de estados como la ilustrada en el diagrama de la Fig. 5, que permite conmutar el estado del robot en función del nivel, o de los cambios de nivel, de las señales medidas por el 
sistema Mindwave. Las variables de entrada que se tomarán en cuenta para hacer evolucionar la máquina de estado son dos: la intensidad de parpadeo (Ip) y el nivel de atención $(\mathrm{Na})$ o el nivel de meditación $(\mathrm{Nm})$. Las variables de salida son: $\mathrm{v}_{\mathrm{l}} \mathrm{y} \mathrm{w}$.

Básicamente la máquina presenta dos estados de movimiento y dos estados de parada. Uno de los estados de movimiento activa el comportamiento ML y otro activa el comportamiento MG. La conmutación entre un comportamiento u otro tiene lugar cuando la Ip es mayor que un determinado umbral $\left(\tau_{\mathrm{pm}}\right)$. De esta manera, controlando la intensidad del parpadeo, el robot se desplaza hacia delante o gira sobre sí mismo. Para mantenerse en ambos estados de movimiento, es necesario que el $\mathrm{Na}$ esté por encima de un determinado valor (Nmin). Con los dos estados de parada se consiguen dos cosas: por un lado, el robot no se moverá si el nivel de atención no está por encima de un nivel estipulado; por otro, sirven de mecanismo de paro, cuando el usuario quiere detener el robot, solo deber modificar su estado de atención.

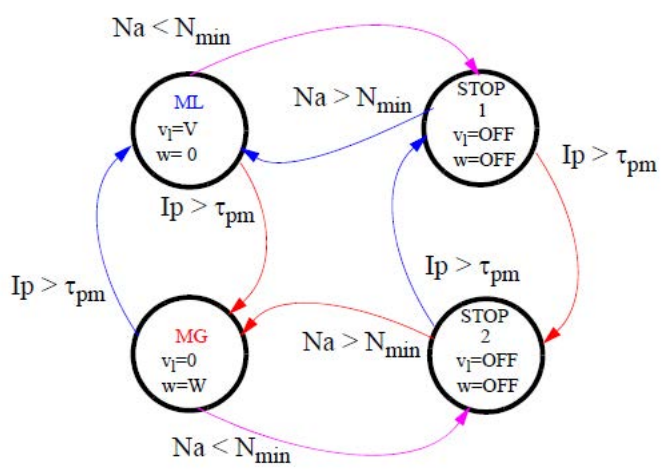

Figura 5: Diagrama de la Máquina de Estados

Adicionalmente, se ha implementado una segunda máquina de estado que se encarga de conmutar el signo de las velocidades, ver Fig. 6.

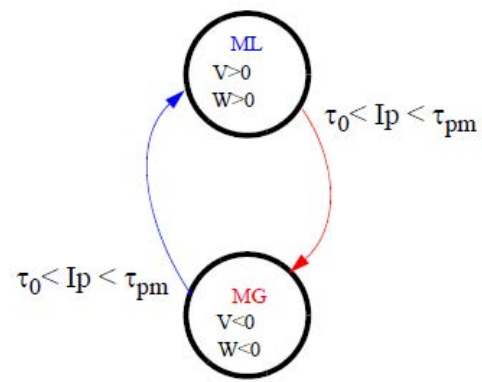

Figura 6: Máquina de Estados para el control de la velocidad

La implementación de esta máquina permite la realización de giros a la derecha y a la izquierda, así como maniobras más complejas con movimiento hacia delante y hacia atrás. La conmutación de estos estados se produce mediante una acción de parpadeo cuya intensidad se encuentra en un intervalo de valores inferior al asociado a la conmutación de comportamientos.

\section{RESULTADOS EXPERIMENTALES}

Para entender la potencialidad de esta aplicación, se han realizado pruebas con diversas personas que no han experimentado antes con el sistema. De hecho, la plataforma tiene como objetivo facilitar su uso a usuarios, que no están familiarizadas ni con el uso del sistema Mindwave, ni con la teleoperación de robots móviles.

Con este fin, antes de interactuar con el robot real, se ha propuesto un plan de adiestramiento que permite manejar el robot con soltura tras un reducido número de sesiones. En primer lugar, se ha utilizado la aplicación desarrollada en Matlab que monitoriza las señales de atención y meditación. Con ella se adiestra al usuario a mantener alto el nivel de ambas señales. En la Fig. 7 se muestra la evolución de las señales cuando el usuario ha adquirido práctica manteniendo un nivel constantemente alto de atención. Puede observarse que, como consecuencia del entrenamiento, la onda de atención representada en este gráfico difiere de la onda representada en el de la Fig. 4, no solo en los valores altos que contiene sino también en que su valor es más regular, sin grandes oscilaciones.

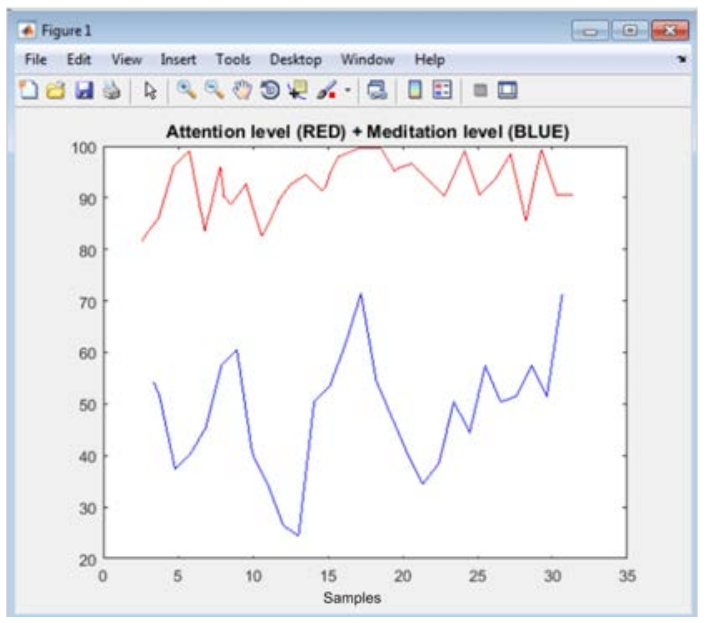

Figura 7: Grafica con un alto el nivel de atención

Un proceso similar se realiza con la intensidad del parpadeo. Además, con esta señal es más fácil adiestrar al usuario para conseguir distintas intensidades, lo que facilitará el desarrollo de la 
habilidad en la realización de transiciones de estados en las dos máquinas descritas anteriormente.

A continuación, los usuarios son entrenados en un escenario virtual. Se trata de otra aplicación desarrollada en Matlab en la que se maneja un robot virtual (representado por un cuadrado en la Fig. 8) para llegar a un punto en concreto (el rojo en la Fig. 8) después de moverse alrededor de varios obstáculos de distinto color.

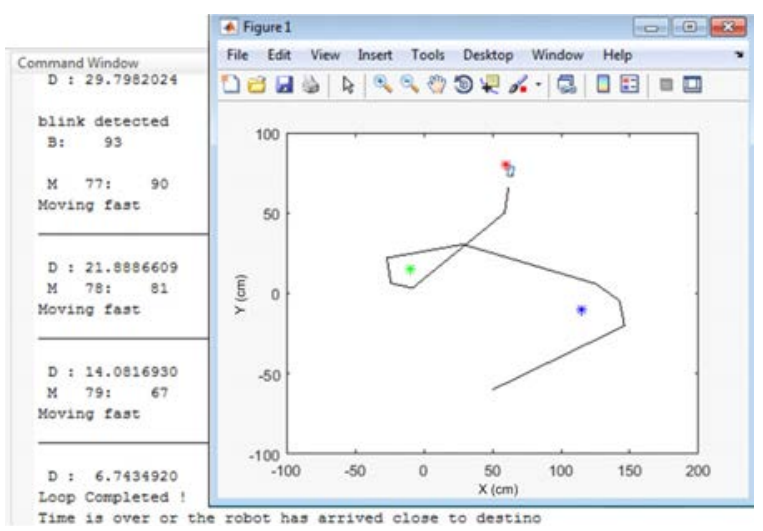

Figura 8: Simulación virtual.

Finalmente, los usuarios se entrenan en la conducción del robot con diferentes escenarios.

El primer escenario real con el que se adiestró a los operadores noveles se muestra en la Fig. 9. Se trata de un pasillo aproximadamente circular, por donde debe circular el robot. Experimentando con distintas personas, el tiempo medio de entrenamiento que llevó realizar con éxito la prueba, fue de aproximadamente 2 horas, incluyendo todo el proceso de entrenamiento antes descrito.

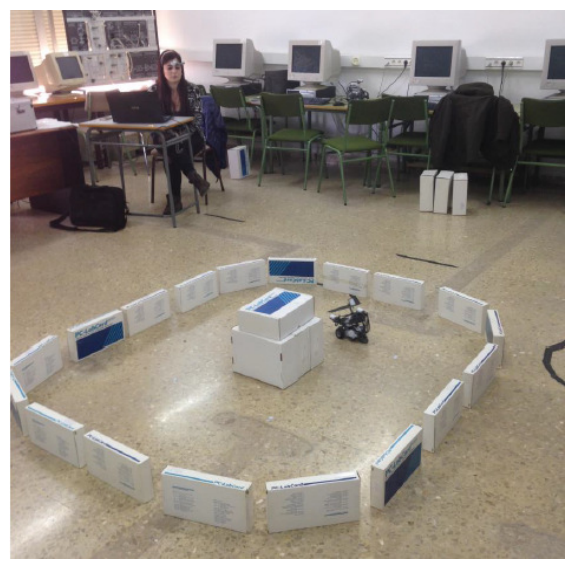

Figura 9: Escenario inicial.

Con posterioridad se realizaron los experimentos con escenarios de mayor complejidad, que a continuación se presentan, que fueron ejecutados con éxito tras un periodo de entrenamiento que abarcó varios días no consecutivos.
En la Fig. 10 se presenta una fotografía del siguiente escenario de adiestramiento. Se trata de un camino con un par de zonas de aparcamiento (A y B) donde el usuario debe ser capaz de mover el robot desde A hasta B sin chocar con las paredes. Se recomendó usar el trazado amarillo de manera que no fuera necesario girar hacia la derecha. El usuario solo tenía que elegir el momento adecuado para parpadear con el objeto de girar a la izquierda 5 veces y llegar a la zona B.

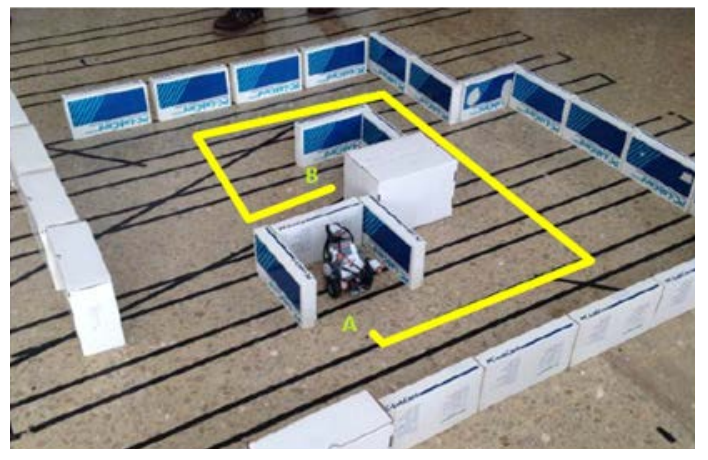

Figura 10: Escenario solo giro a derechas

En la Fig. 11 se muestran varias fotografías tomadas durante la realización de la prueba. Para la ejecución con total éxito de la misma, solo fue necesario un periodo de entrenamiento que abarcó tres días no consecutivos.

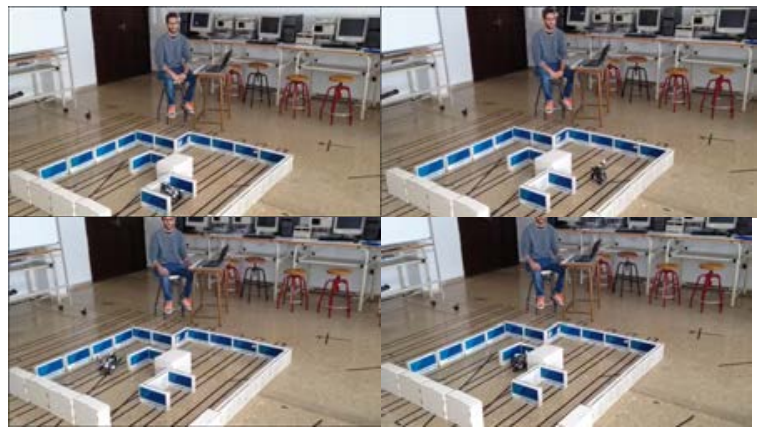

Figura 11: Experimento en el escenario de la Fig. 10.

En la Fig. 12 se ilustra un nuevo trazado donde ya se hace necesario que el robot gire tanto a la izquierda como a la derecha. Para, conseguir la conmutación del signo de la velocidad de giro el usuario debía ser capaz de producir una intensidad de parpadeo que se fijó entre 85 y 169. Para la conmutación entre desplazamiento lineal y giro debía ser capaz de producir una intensidad de parpadeo mayor que 169. En la Fig. 13 se muestran varias fotografías tomadas durante la realización de la prueba. Se necesitó solo 30 minutos para el entrenamiento y ejecución con éxito de este circuito, resultando que la mejor manera de conducir al robot a lo largo de este trazado siguió los siguientes comandos: mover hacia adelante, parpadear entonces ligeramente para girar a la 
izquierda y a continuación mover hacia adelante, luego, parpadear con fuerza para cambiar el valor del signo de la velocidad de giro y parpadear ligeramente, otra vez, para girar a la derecha. Realizando esta operación sucesivamente, se logró que el robot transitara a través del pasillo.

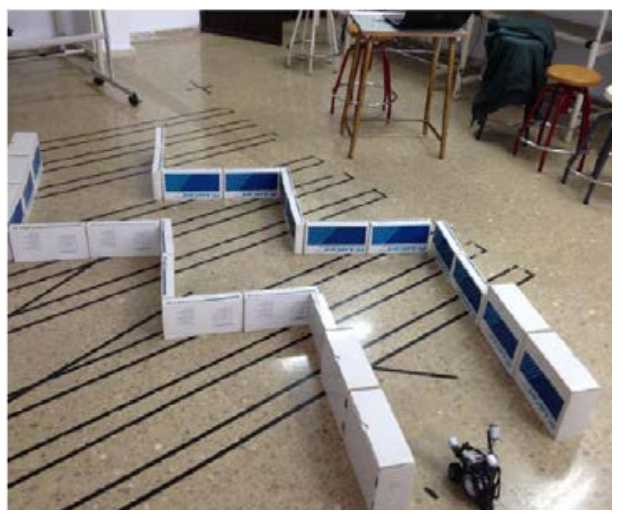

Figura 12: Escenario en Zig-Zag

Todas las operaciones estaban afectadas por un tiempo de retardo, debido al largo trayecto que las señales deben recorrer: primero la adquisición de la onda cerebral por el ICC, la trasmisión al PC, su procesamiento, su visualización y utilización en Matlab, y por último, la transmisión del comando generado al robot

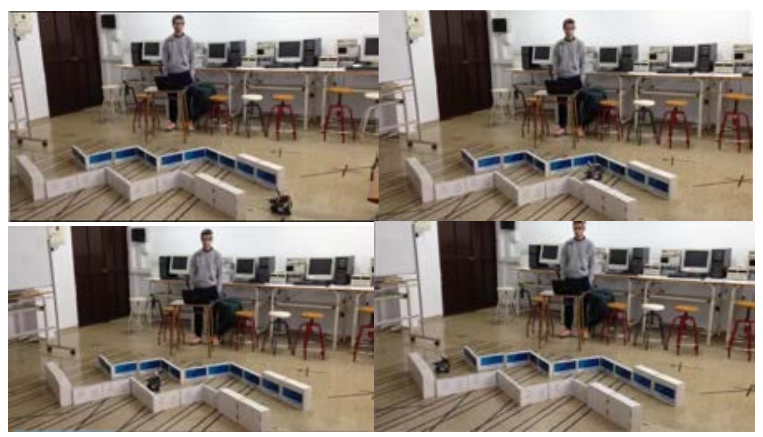

Figura 13: Experimento en el escenario de la Fig. 12.

Este retraso se ha contabilizado en un rango de 1 a 1.5 segundos y principalmente está causado por la conexión bluetooth, y en menor medida, por la generación de las señales para el robot. Por ello el usuario debe entrenarse, además de para controlar la meditación y la intensidad del parpadeo, para tener en cuenta el retraso en la respuesta del robot y prever las acciones.

\section{CONCLUSIONES}

En este artículo se presenta un sistema para teleoperar un robot móvil basado en el uso del ICC Mindwave. La característica diferenciadora de este ICC se encuentra en el bajo número de electrodos, y en su bajo coste. Además, el sistema proporciona una estimación del estado de atención y de la intensidad de parpadeo del usuario. El sistema propuesto utiliza estas señales para implementar un sistema de control basado en dos máquinas de estado. La implementación ha logrado reducir los tiempos de retardo típicos de estos sistemas. De este modo, mediante la estrategia desarrollada, un usuario sin conocimiento previo del sistema es capaz de maniobrar el robot con un tiempo de entrenamiento inferior a dos horas.

\section{Referencias}

[1] Brunner C. et al., (2015) BNCI Horizon 2020: towards a roadmap for the BCI community, Brain-Computer Interfaces, Vol. 2, No. 1, pp. $1-10$.

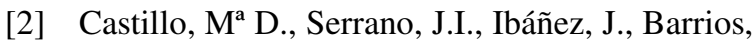
L.J., (2011) Metodología para la Creación de una Interfaz Cerebro-Computador Aplicada a la Identificación de la Intención de Movimiento, Revista Iberoamericana de Automática $e$ Informática Industrial, Vol. 8, no. 2, pp 93-102.

[3] Christoph G., (2010) State of the Art in BCI Research: BCI Award, g.tec medical engineering $\mathrm{GmbH} / \mathrm{Guger}$ Technologies OG, Graz, Austria.

[4] Escolano C., Minguez J., (2011) Sistema de Teleoperación Multi-Robot basado en Interfaz Cerebro-Computador, Revista Iberoamericana de Automática e Informática Industrial, Vol. 8, no. 2, pp 16-23.

[5] Li Zhao, Chuo Li, Shigang Cui, (2007) Service Robot System Based on Brain-computer Interface Technology, Tianjin University of Technology and Education Institute of Semic0onductors, CAS Tianjin, China.

[6] NeuroSky, Inc. (2009), NeuroSky's eSenseTM Meters and Detection of Mental State, White paper disponible on-line en neurosky.com.

[7] Ollero, A. Róbótica; Manipuladores y Robots Móviles. Marcombo (2001).

[8] Sudarsanan K., Dr. S. Sasipriya, (2014) Controlling a Robot Using Brain Waves, UG student final year ECE, Park College of Engineering and Technology, Coimbatore, India.

[9] Toolbox RWTH - for Lego Mindstorms NXT (http://www.mindstorms.rwth-aachen.de/) 